\title{
Plasmablastic multiple myeloma is associated with increased vascular endothelial growth factor immunoexpression
}

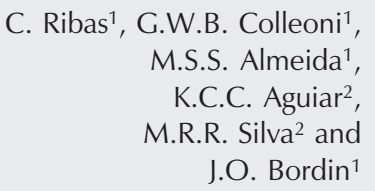

\author{
1Disciplina de Hematologia e Hemoterapia, ${ }^{2}$ Departamento de Patologia, \\ Escola Paulista de Medicina, Universidade Federal de São Paulo, São Paulo, \\ SP, Brasil
}

\author{
Correspondence \\ G. Colleoni \\ Disciplina de Hematologia \\ EPM, UNIFESP \\ Rua Botucatu, 740, 3o andar \\ 04023-900 São Paulo, SP \\ Brasil \\ Fax: +55-11-5571-8806 \\ E-mail: gcolleoni@hemato.epm.br \\ Research supported by CNPq. \\ C. Ribas was partially supported \\ by $\mathrm{CNPq}$. \\ Publication supported by FAPESP.

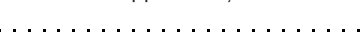

Received October 7, 2004

Accepted May 3, 2005

\begin{abstract}
The biologic basis of the negative prognosis of plasmablastic myeloma is not fully understood. To determine whether histologically aggressive multiple myeloma (MM) is associated with a more angiogenic marrow environment, bone marrow samples from 50 recently diagnosed MM patients were evaluated. Twelve percent (6/50) of patients presented plasmablastic MM, and this feature correlated with moderate/strong intensity of vascular endothelial growth factor staining of plasma cells $(\mathrm{P}=0.036)$. Although plasmablastic MM was not associated with increasing of microvessel density, this new evidence of increased expression of vascular endothelial growth factor on plasmablasts suggests that the adverse prognosis conferred by plasmablastic disease may be due, at least in part, to secretion of this angiogenic cytokine, also suggesting that the subset of MM patients with plasmablastic features may derive particular benefit from antiangiogenic therapies.
\end{abstract}

Key words

- Multiple myeloma

- Angiogenesis

- Vascular endothelial

growth factor

\section{Introduction}

The amount of microvessels in the bone marrow progressively increases along the spectrum of plasma cell disorders and becomes a prominent feature in active multiple myeloma (MM) (1). Some studies have established that MM patients with increased marrow angiogenesis have shorter overall survival $(2,3)$. Vascular endothelial growth factor (VEGF) is one of the most important angiogenic cytokines mediating tumor an- giogenesis (4); assessed by immunohistochemical methods, it has been detected in plasma cells and plasmacytoma samples from most MM patients $(5,6)$.

Based on the hypothesis that the adverse clinical evolution associated with plasmablastic MM $(7,8)$ could be determined by an increase of angiogenesis, we studied the correlation between plasmablastic morphology, microvessel density (MVD) and VEGF immunoexpression in the bone marrow samples of 50 newly diagnosed MM patients. If this 
hypothesis were correct, this subset of MM patients would be particularly suitable for antiangiogenic therapies.

\section{Material and Methods}

\section{Patients}

We studied bone marrow samples from fifty recently diagnosed MM patients, followed at the Multiple Myeloma Outpatient Service of the Discipline of Hematology and Hemotherapy, UNIFESP/EPM, São Paulo, SP, Brazil, between May 2000 and December 2002. Formalin-fixed, paraffin-embedded bone marrow sections from 49 patients were available, and 46 patients had also assessable bone marrow smears. Written informed consent was obtained from all patients and the institution's Ethics Committee approved the study.

\section{Assessment of morphologic features}

Marrow smears were stained with MayGrünwald-Giemsa solution to evaluate plasma cell number and morphology. Bone marrow sections were studied after staining with hematoxylin and eosin, Giemsa and PAS. The disease was classified as plasmablastic or non-plasmablastic according to the classification system of Bartl et al. (9), and the percentage of infiltrating plasma cells (tumor burden) was recorded.

\section{Immunohistochemical studies}

Immunohistochemical reactions were performed using the streptavidin-biotin-peroxidase method and the following monoclonal antibodies: anti-kappa light chain (1:5000; Dako, Carpinteria, CA, USA), antilambda light chain (1:5000; Dako), antiCD34 (1:50; Dako), and anti-VEGF (1:100; Santa Cruz Biotechnology, Santa Cruz, CA, USA). The LSAB detection kit (Dako) was used for antigen visualization. Paraffin sec- tions of placental tissue were included in each batch as positive control for CD34 immunohistochemical staining and sections processed with exclusion of the primary antibody were used as negative control. Sections were counterstained with hematoxylin and examined by light microscopy.

\section{Quantification of microvessels in bone marrow}

Angiogenesis was measured by counting the CD34-labeled intratumoral microvessels (MVD) (1), and the patients were stratified into high-angiogenesis (MVD $\geq 20)$ and lowangiogenesis status (MVD <20) according to a previous study (10). To estimate the MVD, the entire slide was scanned at 100X magnification to locate three areas of maximum microvessels (hot spots) in which the microvessels were counted at $400 \mathrm{X}$. The MVD was reported as the average number of vessels in the three hot spots (1).

\section{Assessment of VEGF expression}

Cases were considered to be positive when $\geq 10 \%$ of plasma cells showed the typical pattern of VEGF expression, evidenced by diffuse cytoplasmic brown staining of variable intensity among individual patients. For the positive cases, VEGF immunoreactivity was examined on the basis of staining intensity (weak and moderate/strong). Given the known VEGF expression by marrow myelo-monocytic and megakaryocytic cells, which invariably showed strong VEGF staining, these cells were used as positive internal controls $(4,5)$.

\section{Statistical analysis}

Associations between nominal variables were tested by the Pearson chi-square test $\left(\chi^{2}\right)$ or by the Fisher exact test, with the level of significance set at 5\%. Statistical analysis was performed using the SPSS 8.0 software. 


\section{Results}

The clinical characteristics of the patients are summarized in Table 1.

\section{Histological characteristics}

Bone marrow biopsies were available for 49 patients. Plasma cell infiltration in marrow samples ranged from 20 to $100 \%$ (median $80 \%)$. According to the classification of Bartl et al. (9), 6 patients (12\%) presented plasmablastic disease, while $43(88 \%)$ did not (Figure 1).

\section{Bone marrow angiogenesis}

Forty-eight CD34-stained marrow sections were available to assess angiogenesis. Median MVD was 25 microvessels per 400 high-power field (range: 3-130). Low angiogenesis (MVD <20) was present in $13(27 \%)$ patients, and high angiogenesis (MVD $\geq 20$ ) was present in 35 patients (73\%).

\section{VEGF expression}

Forty-nine sections were assessable for VEGF analysis. Fourteen (29\%) patients showed VEGF expression in $<10 \%$ positive plasma cells (negative cases), while 35 (71\%) showed VEGF expression in $\geq 10 \%$ positive plasma cells. Among the positive cases, 17
(49\%) displayed a weak intensity signal, while $18(51 \%)$ showed moderate/strong intensity.

\section{Correlations between morphologic feature,} VEGF expression and bone marrow angiogenesis

Plasmablastic morphology did not show an association with tumor burden in bone

Table 1. Clinical characteristics of the 50 patients studied.

\begin{tabular}{lc}
\hline Patients' baseline characteristics & $\mathrm{N}$ \\
\hline Age (years) & \\
Median & 57 \\
Range & $28-83$ \\
Gender & \\
Male & \\
Female & $29(58 \%)$ \\
Salmon-Durie stage & $21(42 \%)$ \\
Ila & \\
IIb & $2(4 \%)$ \\
IIla & $1(2 \%)$ \\
IIlb & $19(38 \%)$ \\
Isotype & $28(56 \%)$ \\
IgG & \\
IgA & $24(48 \%)$ \\
IgM & $10(20 \%)$ \\
Not IgM, IgA, IgG & $1(2 \%)$ \\
Data missing & $12(24 \%)$ \\
Light chain & $3(6 \%)$ \\
Kappa & \\
Lambda & $27(54 \%)$ \\
Not available & $18(36 \%)$ \\
& $5(10 \%)$ \\
\hline
\end{tabular}

Figure 1. Micrographs showing plasmablasts in marrow smears (A), plasmablasts in a marrow biopsy (B), and plasmablasts with strong vascular endothelial growth factor immunostaining in a marrow biopsy (C). Original magnification, 400X. 
marrow biopsies $(\mathrm{P}=0.614)$; also, there was no association with angiogenesis status, as assessed by MVD ( $\mathrm{P}=0.713)$. However, plasmablastic MM significantly correlated with moderate/strong VEGF intensity $(\mathrm{P}=$ 0.036; Table 2).

\section{Discussion}

Plasmablastic morphology is a well-recognized adverse morphologic feature in MM $(7,8)$. Findings from clinical and laboratory studies have suggested that angiogenesis takes part in the pathogenesis of MM (11), and some studies have revealed angiogenesis as a prognostic factor for disease-related survival and as a significant predictor of plasmacytoma progression to MM $(2,3)$.

The goal of this study was to investigate the potential links between plasmablastic morphology and angiogenesis in a series of advanced, recently diagnosed MM patients, hypothesizing that a histologically aggressive disease would also correspond to a more angiogenic bone marrow environment. Assessing VEGF immunoexpression in marrow biopsies in terms of staining intensity, we arbitrarily set $\geq 10 \%$ of positive plasma

Table 2. Correlation between the classification of Bartl et al. (9) and vascular endothelial growth factor intensity and microvessel density in multiple myeloma patients.

\begin{tabular}{lcc}
\hline $\begin{array}{l}\text { Bartl's classification } \\
\text { PC BM (\%) }\end{array}$ & $\begin{array}{c}\text { Plasmablastic } \\
(\mathrm{N})\end{array}$ & $\begin{array}{c}\text { on-plasmablastic } \\
(\mathrm{N})\end{array}$ \\
$<80 \%$ & $2(33 \%)$ & $10(24 \%)$ \\
$\geq 80 \%$ & $4(67 \%)$ & $32(76 \%)$ \\
VEGF intensity* & & $14(33 \%)$ \\
$\quad$ Negative & 0 & $16(37 \%)$ \\
$\quad$ Weak & $1(17 \%)$ & $13(30 \%)$ \\
Moderate/strong & $5(83 \%)$ & \\
Angiogenesis status & & $11(26 \%)$ \\
$\quad$ Low (MVD <20) & $2(33 \%)$ & $31(74 \%)$ \\
High (MVD $\geq 20)$ & $4(67 \%)$ & \\
\hline
\end{tabular}

PC BM = plasma cells in bone marrow (median 80); VEGF = vascular endothelial growth factor; MVD = microvessel density.

*Plasmablastic multiple myeloma significantly correlated with moderate/strong VEGF intensity $(P=0.036)$ cells as the cut-off for considering a case as positive.

Bellamy et al. (4) studied the VEGF mRNA and protein expression in 12 human hematopoietic tumor cell lines and in 16 MM patients. VEGF expression was defined according to the intensity of cytoplasmic staining; immunohistochemical analysis was performed on formalin-fixed, paraffin-embedded bone marrow clots or cores, and plasma cell expression of VEGF was observed in 12 of 16 patients, presenting as diffuse cytoplasmic staining of variable intensity among individual patients. With an expanded cohort of $42 \mathrm{MM}$ patients, using the same immunohistochemistry method, Bellamy (5) was able to observe plasma cell expression of VEGF in the bone marrow of $33(78 \%)$ patients.

We found an interesting association between plasmablastic morphology and moderate/strong VEGF intensity even though we did not detect a correlation between plasmablastic morphology and angiogenesis status. This finding, although limited by the small number of patients with plasmablastic features, suggests that a histologically more aggressive disease may be associated with intensified production of VEGF. Although VEGF expression has been significantly associated with immature morphology in plasmacytomas (6), the correlation in MM has not been previously reported.

Rajkumar et al. (1) analyzed angiogenesis in 400 patients with plasma cell dyscrasias regarding medullary microvessels identified by immunohistochemical staining for CD34. From the relatively benign monoclonal gammopathy of undetermined significance to relapsed MM, the median MVD increased significantly and high-grade angiogenesis found to be progressively more frequent, suggesting that angiogenesis may be related to disease progression.

The high-angiogenesis status shared by $73 \%$ of our patients probably reflects the virtually homogenous group of stage III dis- 
ease, highlighting the association of angiogenesis with advanced MM. Although we did not detect an association between plasma cell percentage and MVD or VEGF, the hot spots were generally found to be closely related to focal areas of tumor infiltration (data not shown), suggesting that autocrine production of angiogenic cytokines was taking place.
In short, our findings suggest that plasmablastic MM is associated with increased production of VEGF and confirm the association of advanced MM with increased angiogenesis. In view of current efforts to study antiangiogenic drugs in the treatment of MM (12), we believe that this subset of MM patients could benefit from these therapies.

\section{References}

1. Rajkumar SV, Mesa RA, Fonseca R et al. (2002). Bone marrow angiogenesis in 400 patients with monoclonal gammopathy of undetermined significance, multiple myeloma, and primary amyloidosis. Clinical Cancer Research, 8: 2210-2216.

2. Pruneri G, Ponzoni M, Ferreri AJM et al. (2002). Microvessel density, a surrogate marker of angiogenesis, is significantly related to survival in multiple myeloma patients. British Journal of Haematology, 118: 817-820.

3. Sezer O, Niemoller K, Eucker J et al. (2000). Bone marrow microvessel density is a prognostic factor for survival in patients with multiple myeloma. Annals of Hematology, 79: 574-577.

4. Bellamy WT, Richter L, Frutiger $Y$ et al. (1999). Expression of vascular endothelial growth factor and its receptors in hematopoietic malignancies. Cancer Research, 59: 728-733.

5. Bellamy WT (2001). Expression of vascular endothelial growth factor and its receptor in multiple myeloma and other hematopoietic malignancies. Seminars in Oncology, 28: 551-559.

6. Paydas S, Zorludemir S, Baslamisli F et al. (2002). Vascular endothelial growth factor (VEGF) expression in plasmacytoma. Leukemia and Lymphoma, 43: 139-143.

7. Greipp PR, Leong T, Bennett JM et al. (1998). Plasmablastic morphology - an independent prognostic factor with clinical and labora- tory correlates: Eastern Cooperative Oncology Group (ECOG) Myeloma Trial E9486 Report. Blood, 91: 2501-2507.

8. Rajkumar SV, Fonseca R, Lacy MQ et al. (1999). Plasmablastic morphology is an independent predictor of poor survival after autologous stem-cell transplantation for multiple myeloma. Journal of Clinical Oncology, 17: 1551-1557.

9. Bartl R, Frisch B, Fateh-Moghadam A et al. (1987). Histologic classification and staging of multiple myeloma. A retrospective and prospective study of 674 cases. American Journal of Clinical Pathology, 87: 342-355.

10. Kumar S, Fonseca R, Dispenzieri A et al. (2003). Prognostic value of angiogenesis in solitary bone plasmacytoma. Blood, 101: 17151717.

11. Vacca A, Ribatti D, Presta M et al. (1999). Bone marrow neovascularization, plasma cell angiogenic potential, and matrix metalloproteinase-2 secretion parallel progression of human multiple myeloma. Blood, 93: 3064-3073.

12. Schey SA, Fields P, Bartlett JB et al. (2004). Phase I study of an immunomodulatory thalidomide analog, CC-4047, in relapsed or refractory multiple myeloma. Journal of Clinical Oncology, 22: 32693276. 